\title{
Entropic long range order in a 3D spin glass model
}

\author{
Maria Chiara Angelini ${ }^{1}$ and Federico Ricci-Tersenghi ${ }^{2}$ \\ 1 Dipartimento di Fisica, Università La Sapienza, \\ P.le A. Moro 5, 00185 Roma, Italy \\ ${ }^{2}$ Dipartimento di Fisica, INFN - Sezione di Roma 1 and CNR - IPCF, UOS di Roma, \\ Università La Sapienza, P.le A. Moro 5, 00185 Roma, Italy
}

\begin{abstract}
We uncover a new kind of entropic long range order in finite dimensional spin glasses. We study the link-diluted version of the Edwards-Anderson spin glass model with bimodal couplings $(J= \pm 1)$ on a 3D lattice. By using exact reduction algorithms, we prove that there exists a region of the phase diagram (at zero temperature and link density low enough), where spins are long range correlated, even if the ground states energy stiffness is null. In other words, in this region twisting the boundary conditions cost no energy, but spins are long range correlated by means of pure entropic effects.
\end{abstract}

PACS numbers: 75.50.Lk 
The low temperature phase of frustrated spin models is a very interesting and debated subject [1]. Especially in models with discrete couplings, lowering the temperature the frustration may produce surprising effects. For example, the classical "order by disorder" effect discovered by Villain et al. [2] shows up in 2D frustrated spin systems, where ground states (GS) have no magnetization, while a spontaneous magnetization is present at any positive temperature smaller than the critical one, $0<T<T_{c}$ (and this is a rather counterintuitive result!). In this case, the explanation is simple: due to the frustration, two subset of GS exist, having ferromagnetic and antiferromagnetic long range order respectively; these GS have exactly the same energy and so, at zero temperature $(T=0)$, they perfectly compensate each other, leading to null global magnetization; nonetheless, at positive temperatures, the energy of the ferromagnetic state is lower than the antiferromagnetic one and a long range (ferromagnetic) order is recovered. This example evidences the importance of exact cancelations at $T=0$ in frustrated models.

Among frustrated models, spin glasses (SGs) [3] have a very complex low temperature phase. Entropy fluctuations in SGs with discrete couplings are known to play an important role and are most probably the main mechanism for making the free-energy spectrum gapless [4].

In this work we study $3 \mathrm{D}$ spin glasses with binary couplings $(J= \pm 1)$ at $T=0$, showing that frustration in SGs generates an effect even more impressive than the one found by Villain et al.: a long range order only due to entropic effects. More precisely, in this entropically ordered SG phase, a typical SG sample has many GS with exactly the same energy, such that, summing over all these GS, no long range order is found in the system at $T=0$. However, at a closer look, all these GS are not really equivalent and taking into account also the entropic contribution to the $T=0$ exact computation, we find that a subset of GS is dominating the Gibbs measure and thus leads to long range order in the system.

In order to explain the entropic long range order with simpler words, we consider a pair of spins, $\sigma_{i}$ and $\sigma_{j}$, at a very large distance, $|i-j| \simeq L$ (being $L$ the system size) and try to estimate their thermodynamic correlation $\left\langle\sigma_{i} \sigma_{j}\right\rangle$ at $T=0$ by computing the probabilities of being parallel or antiparallel, $\mathbb{P}\left[\sigma_{i}= \pm \sigma_{j}\right]$. The method, which is typically employed, computes the GS energy at fixed (relative) values of $\sigma_{i}$ and $\sigma_{j}$ : if the resulting GS energy difference $\left|E_{\mathrm{GS}}\left(\sigma_{i}=\sigma_{j}\right)-E_{\mathrm{GS}}\left(\sigma_{i}=-\sigma_{j}\right)\right|$ (the so-called energy stiffness) does not grow with $L$ the system is believed to have no long range order. But this conclusion is wrong! Indeed, 
even if $E_{\mathrm{GS}}\left(\sigma_{i}=\sigma_{j}\right)=E_{\mathrm{GS}}\left(\sigma_{i}=-\sigma_{j}\right)$, the relative orientation of $\sigma_{i}$ and $\sigma_{j}$ still depends on the number of GS, $\mathcal{N}_{\mathrm{GS}}$, with given values of $\sigma_{i}$ and $\sigma_{j}$ :

$$
\mathbb{P}\left[\sigma_{i}= \pm \sigma_{j}\right] \propto \mathcal{N}_{\mathrm{GS}}\left(\sigma_{i}= \pm \sigma_{j}\right) \propto \exp \left[S_{\mathrm{GS}}\left(\sigma_{i}= \pm \sigma_{j}\right)\right]
$$

where $S_{\mathrm{GS}}$ is the GS entropy. If the entropy difference $\left|S_{\mathrm{GS}}\left(\sigma_{i}=\sigma_{j}\right)-S_{\mathrm{GS}}\left(\sigma_{i}=-\sigma_{j}\right)\right|$ grows with $L$, then $\left|\left\langle\sigma_{i} \sigma_{j}\right\rangle\right| \rightarrow 1$ in the thermodynamical limit and the system shows an entropic long range order (the energy stiffness being null). Please note that the present entropic effect is taking place also at $T=0$, while the Villain's "order by disorder" requires a positive temperature because it is due to an energy difference.

We are going to show, by exact reduction algorithms, that such an entropic long range order exists in SGs with discrete couplings on regular lattices in finite dimensions. We consider a link-diluted 3D Edwards-Anderson model defined by the Hamiltonian $H=$ $-\sum_{<i j>} \sigma_{i} J_{i j} \sigma_{j}$, where the sum is over all the nearest neighbor pairs of a 3D simple cubic lattice of length $L$. The couplings $J_{i j}$ are quenched, independent and identically distributed random variables extracted from the distribution

$$
P_{J}(J)=(1-p) \delta(J)+\frac{p}{2}[\delta(J-1)+\delta(J+1)],
$$

where $p \in[0,1]$ is the density of non-zero couplings.

This model has a critical line in the $(p, T)$ plane that separates the paramagnetic phase from the SG phase. It was already shown by Bray and Feng [5] that, while in a model with a continuous couplings distribution this critical line ends for $T=0$ at the geometric link percolation threshold $p_{c}$ [7], for discrete couplings the paramagnetic phase does extend beyond $p_{c}$, because of exact cancellations between positive and negative couplings. Let us call $p_{\mathrm{SG}}$ the critical value separating the paramagnetic from the $\mathrm{SG}$ phase at $T=0$. A tentative estimation of $p_{\mathrm{SG}}$ has been provided by Boettcher [6] by considering the "defect" energy $\Delta E_{\mathrm{GS}}$ between the GS energies obtained by swapping between periodic and antiperiodic boundary conditions along one direction. He found that for $p>p^{*}=0.272(1)$ the variance of $\Delta E_{\mathrm{GS}}$ grows with $L$ (the mean being null by symmetry) thus leading to a SG long range order. After the work of Boettcher the threshold $p^{*}$ has been identified with $p_{\mathrm{SG}}$, but this is not generally true (as we are going to show now). In general only the inequality $p_{\mathrm{SG}} \leq p^{*}$ holds. Recently in Ref. [8] the same model has been solved exactly on the hierarchical lattice, showing that $T=0$ computations can lead to misleading results. 
Indeed, while at $T=0$ the model shows a phase transition at $p^{*}$, the exact solution at positive temperatures predicts a critical line in the $(p, T)$ plane ending in $\left(p_{\mathrm{SG}}, 0\right)$, with $p_{c}<p_{\mathrm{SG}}<p^{*}$ (strict inequalities hold). The right critical point $p_{\mathrm{SG}}$ is clearly sensitive to entropic effects, that are neglected in the computation of $p^{*}$. The determination of the $p_{\mathrm{SG}}$ value can be done by simply considering first order corrections in temperature to the $T=0$ computations. Thus, in the rest of the paper, we are going to work in this $T=0^{+}$limit.

On a 3D cubic lattice the model can not be solved exactly and Monte Carlo methods are very inefficient at low temperatures. To determine the right critical point $p_{\mathrm{SG}}$, we are going to apply some exact decimation rules that reduce the system to a much smaller size, which can be then easily solved by numerical methods.

We consider periodic boundary conditions in $x$ and $y$ directions, while spins in $z=0$ and $z=L-1$ are linked respectively to two different external spins, with quenched, independent and identically distributed random couplings extracted from the distribution in Eq. (11). The addition of these external spins does not modify the thermodynamic limit but it is very useful: to check for percolation will be enough to find a path of non-zero couplings between these two external spins, while to check for the presence of long range order one can just measure the correlation between these two spins. So, in general, one will be satisfied with the computation of the effective coupling between the two external spins.

Given that the model is link diluted, we can eliminate recursively weakly connected spins, generalizing what was done in Refs. [6, 8]. In the original model couplings are Tindependent, but, by decimating spins, effective couplings are created whose intensity will depend on temperature. If we want to find entropic effects, the first order correcting term in $T$ can not be neglected, even studying the system in the $T=0$ limit. For infinitesimal $T$, we can write an effective coupling as $J=\operatorname{sign}(I)(|I|-T K)$ if $I \neq 0$ or $J=T K$ if $I=0$, where $I$ and $K$ are the energetic and entropic coupling respectively. The choice for the relative sign is dictated by the fact that thermal fluctuations decrease the coupling intensity. Spins and bonds are decimated using the following 5 rules.

R1 A zero- or one-connected spin is eliminated.

R2 A two-connected spin $\sigma$ is eliminated and an effective coupling $J_{12}$ is created between the two neighboring spins, $\sigma_{1}$ and $\sigma_{2}$, satisfying the equation

$$
\sum_{\sigma= \pm 1} e^{\left(J_{1} \sigma \sigma_{1}+J_{2} \sigma \sigma_{2}\right) / T} \equiv A e^{J_{12} \sigma_{1} \sigma_{2} / T}
$$


for any choice of $\sigma_{1}$ and $\sigma_{2}$. Expanding at first order in $T$ the two members, we have for the energetic component

$$
I_{12}=\frac{1}{2}\left(\left|I_{1}+I_{2}\right|-\left|I_{1}-I_{2}\right|\right)
$$

and for the entropic component

$$
\begin{array}{rll}
K_{12}=K_{1} & \text { if } & \left|I_{1}\right|<\left|I_{2}\right|, \\
e^{2 K_{12}}=e^{2 K_{1}}+e^{2 K_{2}} & \text { if } & \left|I_{1}\right|=\left|I_{2}\right| \neq 0, \\
\tanh \left(K_{12}\right)=\tanh \left(K_{1}\right) \tanh \left(K_{2}\right) & \text { if } & I_{1}=I_{2}=0 .
\end{array}
$$

R3 Two bonds $J_{i j}^{1}$ and $J_{i j}^{2}$ between two spins $i$ and $j$ can be replaced by an effective coupling $J_{i j}$ with components $I_{i j}=I_{i j}^{1}+I_{i j}^{2}$ and $K_{i j}=K_{i j}^{1}+K_{i j}^{2}$.

$\mathbf{R} 4$ A three-connected spin $\sigma$ is eliminated and effective couplings are created between the three neighboring spins $\sigma_{1}, \sigma_{2}$ and $\sigma_{3}$, satisfying the equation

$$
\sum_{\sigma= \pm 1} e^{\frac{J_{1} \sigma \sigma_{1}+J_{2} \sigma \sigma_{2}+J_{3} \sigma \sigma_{3}}{T}} \equiv A e^{\frac{J_{12} \sigma_{1} \sigma_{2}+J_{23} \sigma_{2} \sigma_{3}+J_{31} \sigma_{3} \sigma_{1}}{T}}
$$

for any choice of $\sigma_{1}, \sigma_{2}$ and $\sigma_{3}$. Expanding at first order in $T$ the two members, and introducing the couplings $\widetilde{J}_{0}=J_{1}+J_{2}+J_{3}$ and $\widetilde{J}_{k}=\widetilde{J}_{0}-2 J_{k}$ with $k=1,2,3$, we get for the energetic components

$$
\begin{aligned}
& I_{12}=\frac{1}{4}\left(\left|\widetilde{I}_{0}\right|-\left|\widetilde{I}_{1}\right|-\left|\widetilde{I}_{2}\right|+\left|\widetilde{I}_{3}\right|\right), \\
& I_{13}=\frac{1}{4}\left(\left|\widetilde{I}_{0}\right|-\left|\widetilde{I}_{1}\right|+\left|\widetilde{I}_{2}\right|-\left|\widetilde{I}_{3}\right|\right), \\
& I_{23}=\frac{1}{4}\left(\left|\widetilde{I}_{0}\right|+\left|\widetilde{I}_{1}\right|-\left|\widetilde{I}_{2}\right|-\left|\widetilde{I}_{3}\right|\right),
\end{aligned}
$$

and for the entropic components

$$
\begin{aligned}
& K_{12}=\frac{1}{4}\left(f\left(\widetilde{J}_{0}\right)-f\left(\widetilde{J}_{1}\right)-f\left(\widetilde{J}_{2}\right)+f\left(\widetilde{J}_{3}\right)\right), \\
& K_{13}=\frac{1}{4}\left(f\left(\widetilde{J}_{0}\right)-f\left(\widetilde{J}_{1}\right)+f\left(\widetilde{J}_{2}\right)+f\left(\widetilde{J}_{3}\right)\right), \\
& K_{23}=\frac{1}{4}\left(f\left(\widetilde{J}_{0}\right)+f\left(\widetilde{J}_{1}\right)-f\left(\widetilde{J}_{2}\right)-f\left(\widetilde{J}_{3}\right)\right),
\end{aligned}
$$

where $f(J)=|K|+\ln \left(1+e^{-2|K|}\right)$ if $I=0$ and $f(J)=\operatorname{sign}(I) K$ if $I \neq 0$.

R5 A spin $\sigma$ of any connectivity is eliminated if the number $n_{I}$ of its couplings with a nonzero energetic component $(I \neq 0)$ does not exceed three $\left(n_{I} \leq 3\right)$. If $i, j=1, \ldots, n_{I}$ index the spins connected to $\sigma$ by couplings with $I \neq 0$, and if $k$ indexes the other neighbours (for 
which $I_{k}=0$ ), then the new couplings $J_{i j}$ are computed following previous rules, while the new couplings $J_{i k}=\operatorname{sign}\left(J_{i}\right) J_{k}$, i.e. $I_{i k}=0$ and $K_{i k}=\operatorname{sign}\left(I_{i}\right) K_{k}$.

We have applied recursively the above five rules in the order they are listed: i.e., at each decimation step, we try to use rule R1, and, only if it does not apply, we try to use rule R2, and if it does not apply, we try to use rule R3, and so on. The decimation process stops when it reaches a reduced graph where none of the above five rules can be applied. This reduced graph does depend on the order the above rules are applied (because rules R4 and R5 increase the degree of neighboring spins), and the order we have chosen is the one producing the smallest reduced graph.

If the couplings have a discrete spectrum then rule R3 may produce exact cancellations, thus leading to null effective couplings: this is the reason why $p_{\mathrm{SG}}>p_{c}$ holds in general for models with discrete couplings. Applying the above rules recursively it is possible that, starting with only energetic couplings, the final effective coupling has only the entropic component (the energetic one being null). In this situation it is clear that entropic couplings are essential even in the $T=0$ limit.

First of all we study percolation properties of the networks of $I$ and $K$ effective couplings that result from the recursive application of the above rules to all bond and spins (except the external ones, that we want to keep). We are mainly interested in the percolation thresholds, $p_{c}^{I}$ and $p_{c}^{K}$, for the energetic and the entropic components. These percolation thresholds do depend on the set of reduction rules and increase if more rules are used 1 . In Fig. 1 we show the percolation probabilities of the networks of $I$ and $K$ effective couplings for many different lattice sizes as a function of the link density $p$. By studying the crossing points $p_{L_{1}, L_{2}}$ of these probabilities for sizes $L_{1}$ and $L_{2}=r L_{1}$ with fixed $r$ (we use $r=3 / 4,3 / 2,2$ ) we have been able to estimate the percolation thresholds $p_{c}^{I}$ and $p_{c}^{K}$ through fits including the first scaling correction [9]: $p_{L, r L}=p_{c}+A_{r} L^{-1 / \nu-\omega}$, as shown in the inset of Fig. 11. The resulting values are $p_{c}^{I}=0.26475(10)$ and $p_{c}^{K}=0.25161(5)$. The value of $p_{c}^{I}$ is correctly lower than $p^{*}=0.272(1)$, the threshold value where a positive energy stiffness emerges: the fact that $p_{c}^{I}$ and $p^{*}$ in general differ can be easily understood by considering the 2D EA model, which is clearly percolating, but has negative energy stiffness. Moreover $p_{c}^{K}$ is lower than $p_{c}^{I}$ because the applied decimation rules leave the energetic component rational, while the

${ }^{1}$ The 5 rules that we use are all those that keep the interactions pairwise. Indeed decimating a 4-connected spin would produce a 4-spins effective interaction. 


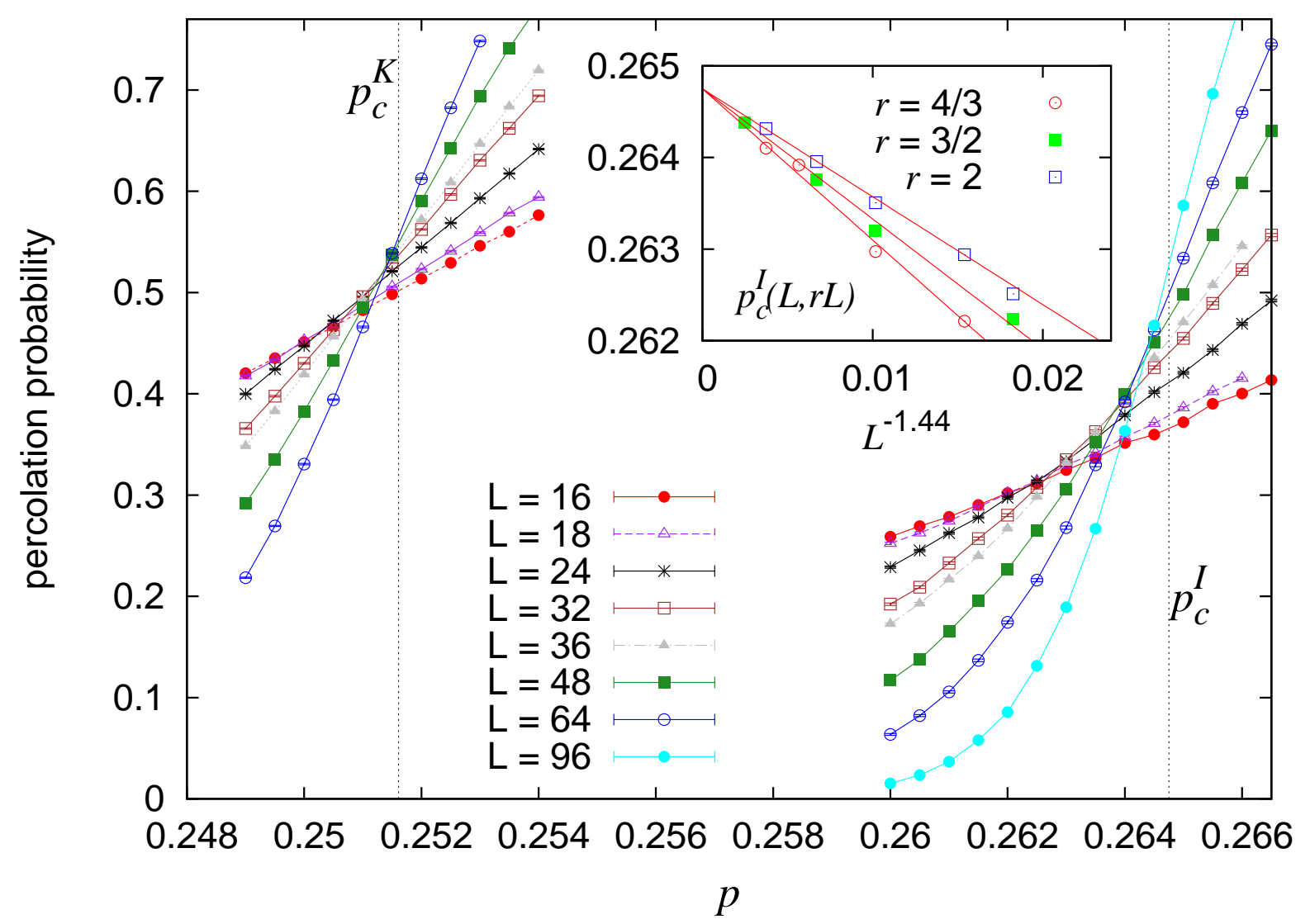

FIG. 1. Percolation probability for different lattice sizes $L$ as a function of the link density $p$ for the energetic (right) and the entropic part (left) of the effective couplings. Inset: the infinite volume extrapolation for $p_{c}^{I}$.

entropic one may become real, thus leading to much less exact cancellations. Please note that $p_{c}^{K}$ provides a lower bound to $p_{\mathrm{SG}}$ given that geometrical percolation of the effective couplings is a necessary, but not sufficient, condition to have SG long range order.

In the thermodynamic limit, for densities smaller than $p_{c}^{I}$, the energetic component $I$ is not percolating and can not induce any long range order. Therefore, in the link density region $p_{c}^{K}<p<p_{c}^{I}$ an eventual thermodynamic phase transition can be due solely to entropic effects.

To search for such an entropic phase transition, we further reduce the decimated graph. For $p_{c}^{K}<p<p_{c}^{I}$, with high probability in the large $L$ limit, the decimated graph is percolating solely in $K$, while the $I$ couplings form clusters of finite size (similarly to what happens in standard percolation below $p_{c}$ ). An example of the resulting graph after the decimation 

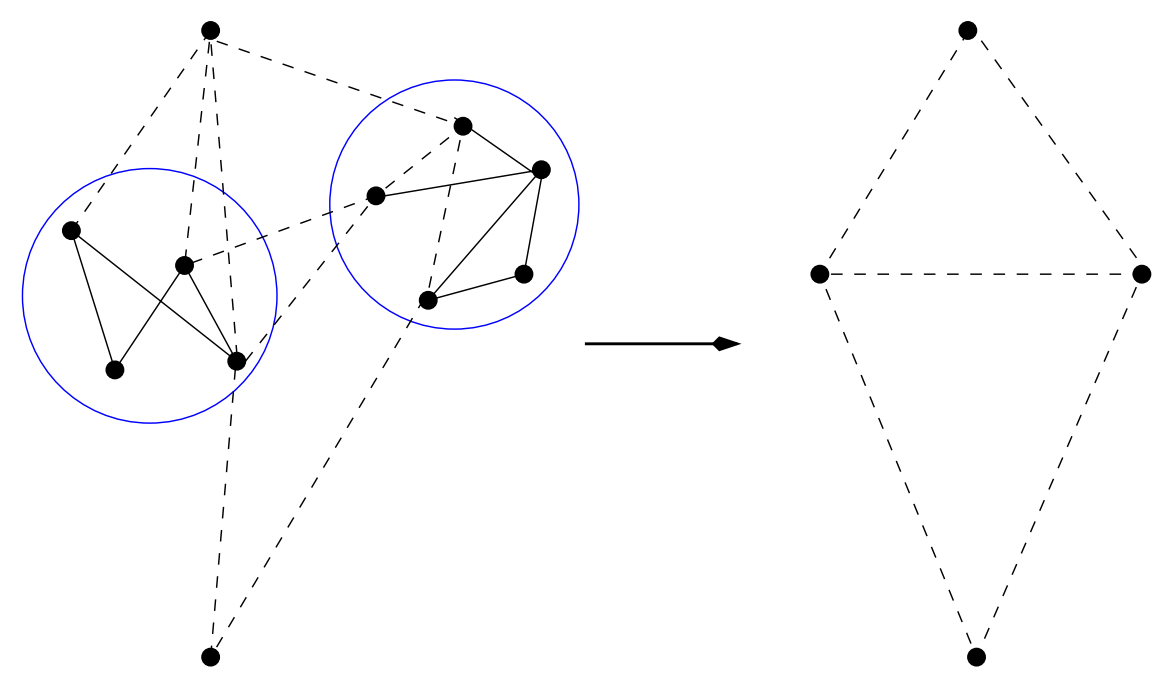

FIG. 2. On the left we show an example of the system after the decimation. Full lines represent couplings with $I \neq 0$, while dashed lines represent couplings with $I=0$. The two $I$-clusters are enclosed in circles and are connected only by purely entropic couplings. On the right, the system is mapped on a Potts model where each variable represents an $I$-cluster. These Potts variables are connected by effective entropic couplings taking into account all the interactions originally connecting the $I$-clusters. Please note that our decimation rules always produce a reduced system with degrees not smaller than 4, but here we have drawn fewer lines for the sake of figure readability.

process is shown on the left side of Fig. 2, where full (resp. dashed) lines represent couplings with (resp. without) a non-null energetic component $I$. The two circles represent what we call $I$-clusters, that is groups of spins connected by couplings with a non-null energetic component $I$ (note, however, that inside these $I$-clusters also couplings with only the entropic component $K$ may exist, as in the rightmost circle in Fig. (21). The connections between any two different $I$-clusters have only entropic components.

Our idea is to map the original problem to a smaller and simpler one, where the variables are the $I$-clusters, that interact only through entropic couplings, as in the right side of Fig. 2

Given that we are interested in the $T=0$ limit, each $I$-cluster must be in a ground state (GS) configuration. So, for each $I$-cluster $\mathcal{C}$, we compute with an exact branch\&bound algorithm all its $\mathcal{N}_{\mathcal{C}}$ GSs. We introduce then a Potts variable $\tau_{\mathcal{C}}$ for that $I$-cluster, taking values in $\left[1, \mathcal{N}_{\mathcal{C}}\right]$. We call $\left\{\sigma_{i}^{\mathcal{C}}\left(\tau_{\mathcal{C}}\right)\right\}$ the GS configurations of the $\mathcal{C}$ cluster.

Working at $T=0$, the GSs are calculated by taking into account solely the energetic component $I$ of the couplings. Afterward we consider also the entropic components $K$, that 
give rise to two different interacting terms. $K$ bonds connecting two spins in the same I-cluster produce a self-interaction term

$$
E^{\mathcal{C}}\left(\tau_{\mathcal{C}}\right)=\sum_{i, j \in \mathcal{C}} K_{i j} \sigma_{i}^{\mathcal{C}}\left(\tau_{\mathcal{C}}\right) \sigma_{j}^{\mathcal{C}}\left(\tau_{\mathcal{C}}\right)
$$

This quantity may bias the choice among degenerate GSs even in the $T=0$ limit. In the new Potts model, it can be interpreted like an external field acting on the Potts variable $\tau_{\mathcal{C}}$ that may bias its value.

$K$ bonds connecting spins in different $I$-clusters generate the interaction between the Potts variables. This interaction depends on the configuration of both clusters, and so must be represented as a matrix

$$
M^{\mathcal{C}, \mathcal{C}^{\prime}}\left(\tau_{\mathcal{C}}, \tau_{\mathcal{C}^{\prime}}\right)=\sum_{i \in \mathcal{C}, j \in \mathcal{C}^{\prime}} K_{i j} \sigma_{i}^{\mathcal{C}}\left(\tau_{\mathcal{C}}\right) \sigma_{j}^{\mathcal{C}^{\prime}}\left(\tau_{\mathcal{C}^{\prime}}\right)
$$

The Gibbs-Boltzmann measure for the reduced Potts model is then

$$
\mu\left(\left\{\tau_{\mathcal{C}}\right\}\right) \propto \exp \left[\sum_{\mathcal{C}, \mathcal{C}^{\prime}} M^{\mathcal{C}, \mathcal{C}^{\prime}}\left(\tau_{\mathcal{C}}, \tau_{\mathcal{C}^{\prime}}\right)+\sum_{\mathcal{C}} E^{\mathcal{C}}\left(\tau_{\mathcal{C}}\right)\right]
$$

It is important to note that this measure does not depend on the temperature, because entropic couplings have a linear dependence on $T$ that cancels the $1 / T$ term in the Boltzmann factor. The Potts measure in Eq. (8) is an exact effective description of the original SG model at temperature $T=0^{+}$, having much less variables and a smaller complexity with respect to the original model.

In order to locate a possible SG transition, we compute the correlation between the external spins under the measure $\mu$ in Eq. (8). If the effective Potts model has a linear topology, namely each variable has at most two neighbors, we solve it exactly by the transfer matrix method (the probability $P$ to have these "linear" systems is rather high: e.g., around the critical density $p_{\mathrm{SG}}, P>0.9$ for $L \leq 24, P \simeq 0.7$ for $L=32, P \simeq 0.6$ for $L=36$ and $P \simeq 0.2$ for $L=48$ ). Otherwise we use a Metropolis Monte Carlo method to sample the measure in Eq.(8) , and the equilibration of the Markov chain is not an issue given the small number of variables. Since the Gibbs-Boltzmann measure in Eq. (8) does not depend on temperature, one can think of it as that of a Potts model at $\beta=1$. Thus, for equilibrating the corresponding Markov chain, we perform a simulated annealing from $\beta=0$ to $\beta=1$, with steps $\Delta \beta=0.1$ and different cooling rates $(100,300$ and 1000 Monte Carlo steps per 


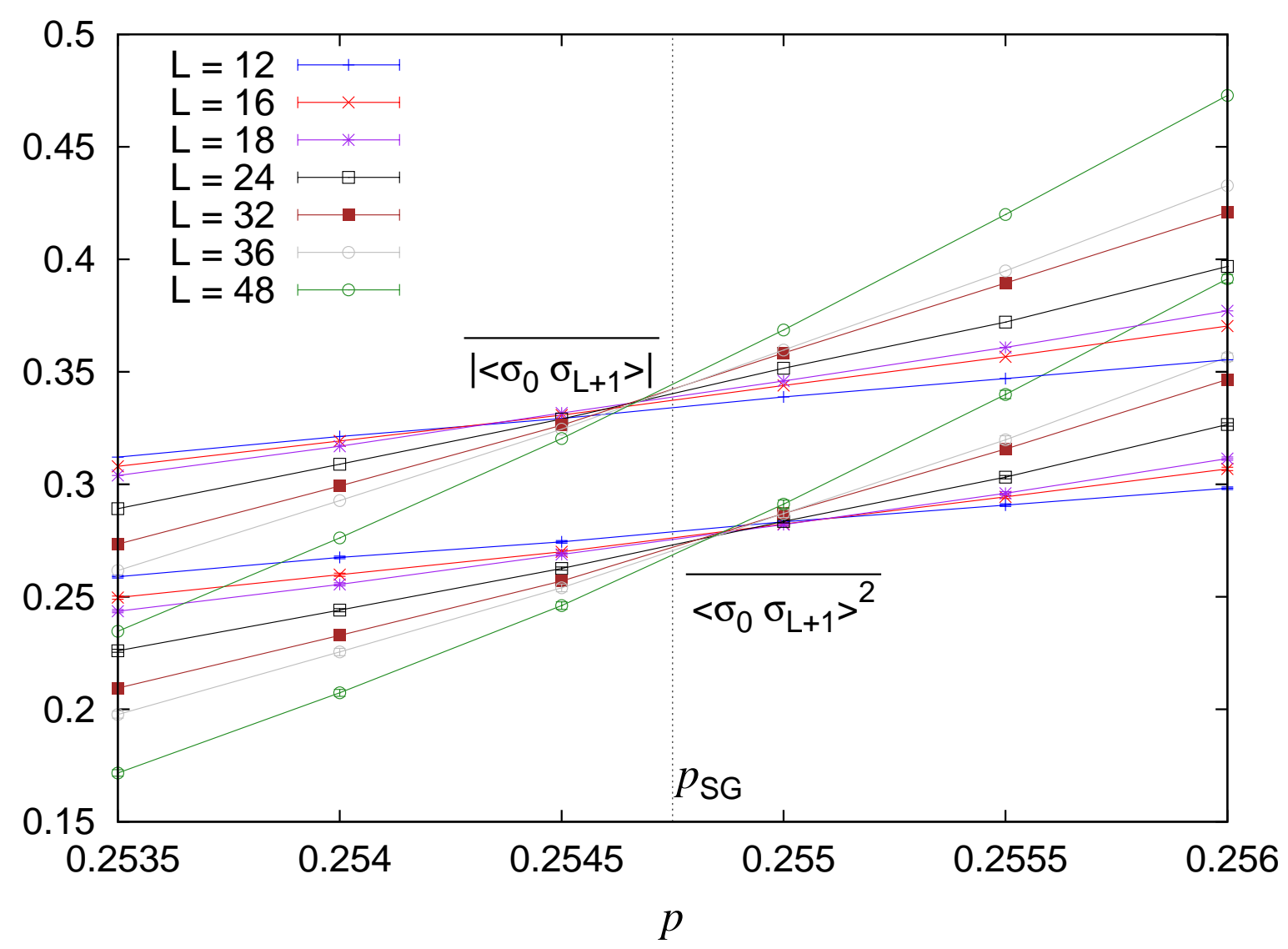

FIG. 3. Average of square (below) and absolute value (above) of correlations at distance $L$, for different lattice sizes, as a function of link density $p$. Errors are not larger than symbols.

temperature). We checked that the average of the interesting quantities, like the correlations, does not dependend on the cooling rate.

For the very few samples that show percolation in the energetic components, we assume a correlation between external spins equal to 1 . This approximation makes no error in the thermodynamical limit as long as $p<p_{c}^{I}$.

Being interested in a SG long range order, we show in Fig. [3 the average over the samples of the square and of the absolute value of the correlation between the external spins (which are at distance $L$ in the original model) as a function of the link density $p$. This quantity should decrease with $L$ in a paramagnetic phase, while it should grow with $L$ if a SG long range order is present: thus the crossing point of the curves in Fig. 3 roughly identifies the critical density $p_{\mathrm{SG}}$. Our best estimation for $p_{\mathrm{SG}}$ has been obtained by the finite-size analysis of the crossing points of the correlations measured in systems of sizes $L$ and $s L$, that should 


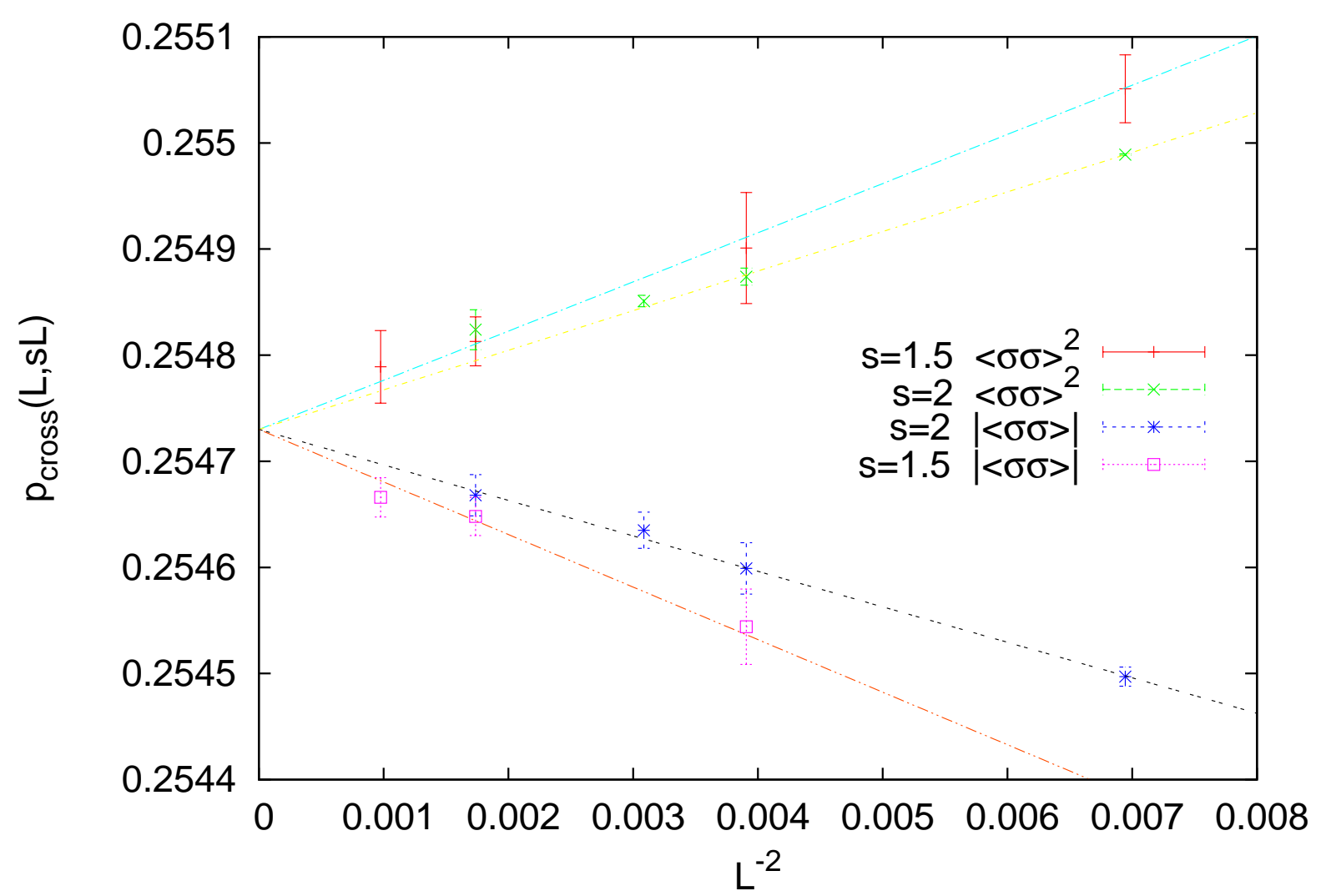

FIG. 4. Crossing points of data shown in Fig. 3 with sizes $L$ and $s L$ as a function of $L^{-2}$.

scale as

$$
p_{\text {cross }}(L, s L)=p_{\mathrm{SG}}+B_{s} L^{-1 / \nu-\omega} \text {. }
$$

In Fig. 4 we show the values of $p_{\text {cross }}$ obtained with $s=1.5$ and $s=2$, together with the best fits. In the abscissa we have used the scaling variable $L^{-2}$ that provides the best joint fit to all the data shown in the figure. However the uncertainty on this scaling exponent is large given the very small spread of $p_{\text {cross }}$ around $p_{\mathrm{SG}}$ for the sizes we have studied. Our final estimation for the $\mathrm{SG}$ critical threshold is $p_{\mathrm{SG}}=0.25473(3)$.

By studying the slopes of the data shown in Fig. 3 at the critical point $p_{\mathrm{SG}}$ as a function of the system size we have been able to obtain an estimation of the exponent $\nu$ controlling the shrinking of the critical region and we get $\nu=0.9(1)$. This value for the $\nu$ exponent does not coincide with the one measured at criticality for the undiluted $(p=1)$ or weakly diluted ( $p=0.45)$ EA model, which is $\nu_{T}=2.2$ (the subscript $T$ should remind us that this exponent is related to the shrinking of the critical region in temperature). However a 


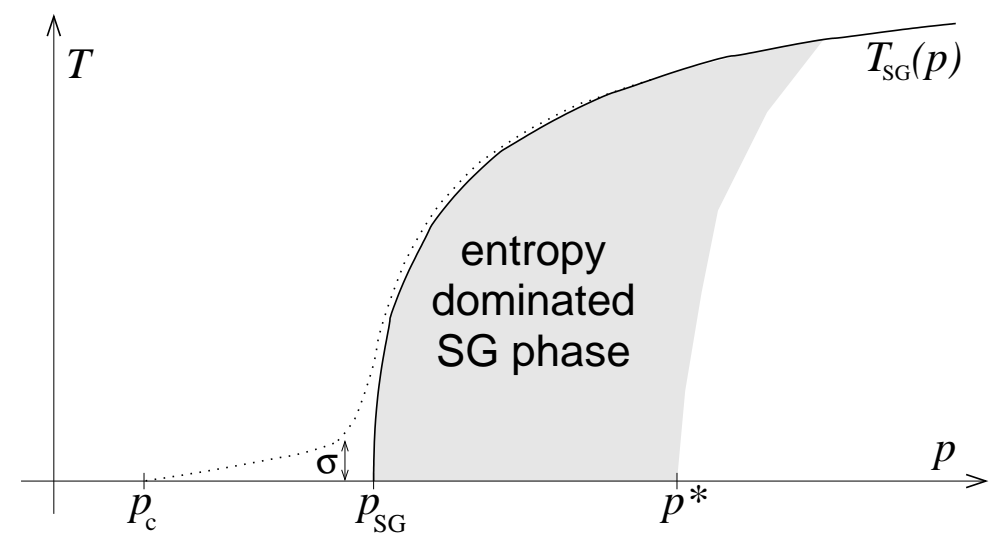

FIG. 5. A schematic phase diagram in the $(p, T)$ plane, showing that entropic long range order must exists also at positive temperatures. The dotted curve is the critical line of a model with quasi-discrete coupling.

simple argument gives the connection between the two exponents: if the critical line close to the $T=0$ fixed point behaves like $T_{\mathrm{SG}}(p) \propto\left(p-p_{\mathrm{SG}}\right)^{\phi}$, then $1 / \nu_{T}=\phi / \nu$. Our results thus suggest a value $\phi \simeq 0.4$ for the shape of the critical line.

We have shown that in 3D spin glasses frustration and coupling discreteness may induce an entropic long range order: in this phase the energy stiffness is zero (i.e. boundary conditions can be changed at no energy cost), but the states with largest entropy dominate the Gibbs measure. This dramatic effect of entropic contributions to the Gibbs states has been extensively studied in mean-field models of spin glasses with finite connectivity at $T=0$, especially in the contest of random constraint satisfaction problems [10 12]. However in the present work we have proved the existence of such an entropic phase in a 3D spin glass model. Moreover this entropy dominated $S G$ phase should persist also at positive temperatures as long as $p \lesssim p^{*}$ and the energy stiffness is null (see Fig. [5).

One may question that perfectly discrete couplings are difficult to find in Nature. Nonetheless if one considers a model with quasi-discrete couplings (e.g. integer values plus a small Gaussian term of variance $\sigma^{2} \ll 1$ ) the critical line looks like the dotted curve in Fig. 5: it mainly follows the critical line of the corresponding model with discrete couplings and only for $T \lesssim \sigma$ moves towards $p_{c}$. It is clear that the identification of such a critical line is based on the correct estimation of $p_{\mathrm{SG}}$ in the model with discrete couplings.

One more comment about the generality of our results, regards what happens when couplings have a ferromagnetic bias. Indeed perfectly symmetric coupling (i.e. with a null 


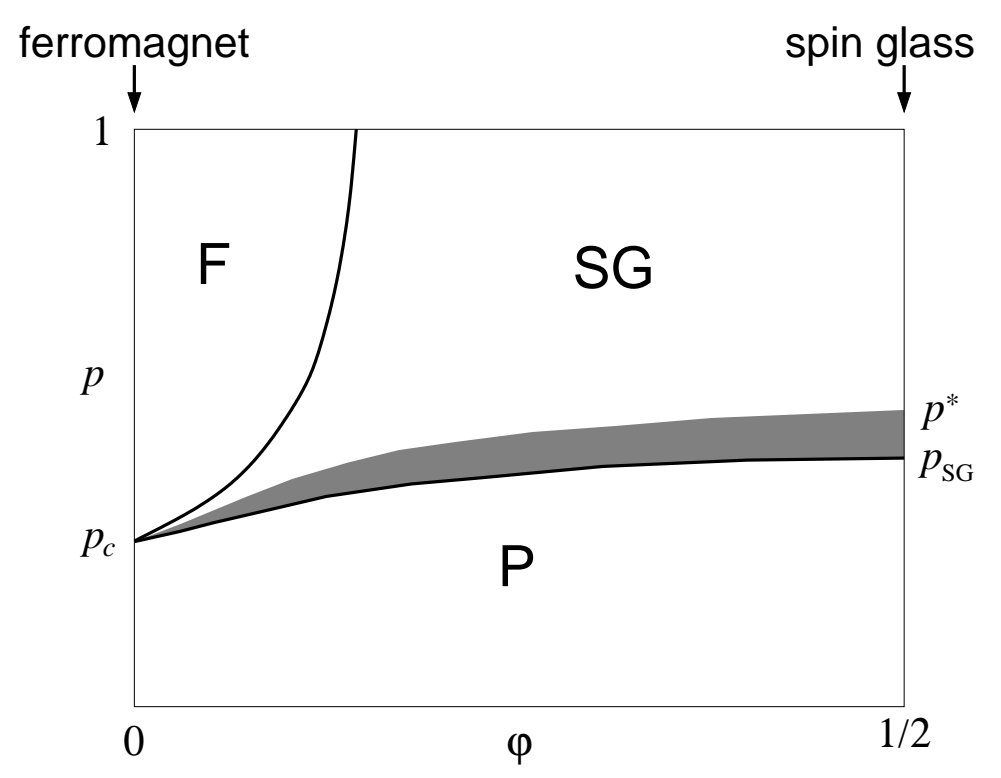

FIG. 6. Conjectured phase diagram at $T=0^{+}$by varying the level of frustration $\varphi$ in the model. The entropically long ranged phase should exist for any frustrated model, $\varphi>0$, with discrete energy levels.

mean, $\overline{J_{i j}}=0$ ) are again difficult to find in Nature, and it is important to check whether the entropic long range order is stable with respect to the addition of a ferromagnetic bias in the couplings. The answer is contained in the pictorial phase diagram shown in Fig. 6, where the link density $p$ is reported as a function of some degree of frustration $\varphi$. A quantitative measure for $\varphi$ on a regular lattice can be, for example, the fraction of frustrated elementary plaquettes: for $\varphi=0$ we have a pure ferromagnetic model, while for $\varphi=1 / 2$ we have the spin glass model studied in this work. In this phase diagram, the addition of a ferromagnetic bias in the couplings corresponds to reducing the value of $\varphi$ with respect to the value $\varphi=1 / 2$ it takes in a spin glass model with symmetrically distributed couplings. The phase diagram shown pictorially in Fig. 6 contains, in general, three different phases: a paramagnetic one $(\mathrm{P})$, a spin glass one $(\mathrm{SG})$ and a ferromagnetic one $(\mathrm{F})$. Moreover, along the SG-F boundary a mixed phase can exist [13], containing a diverging number of states with a non-null magnetization (but here we do not want to enter the long-standing debate about the nature of the spin glass phase in 3D models). In Fig. 6 the gray region is our educated guess about the location of the entropically long range ordered phase: in other words we conjecture the presence of such a phase in any frustrated model $(\varphi>0)$ with discretized energy levels. 
An important comment regards the implications of our results on the studies of the low temperature phase of SG models made by means of GS computations. In these numerical studies one or few GS are usually computed per sample, under different boundary conditions, and only the GS energies are considered. Unfortunately this kind of study is not able to identify the entropic long range order. In light of our results, this kind of numerical studies should be modified either considering the first order correction in temperature when decimating the variables, either computing many (or all) GS per sample, such as to identify the state which is entropically dominating. Some steps in this direction have been already taken in [14], where it has been recognized that a correct estimation of the GS clusters entropy is necessary to extend predictions at positive temperatures.

Last, but not least, the present best estimation for the lower critical dimension in SGs, $d_{L} \simeq 2.5$, is based on GS energy stiffness computations [6], which ignore entropic effects. Most probably this result need to be modified to a lower value due to the entropic long range order.

[1] H.T. Diep, Frustrated Spin Systems (World Scientific, Singapore, 2005).

[2] J. Villain et al., J. Phys. (Paris) 41, 1263 (1980).

[3] K.H. Fischer and J. Hertz, Spin glasses (Cambridge University Press, 1993). A.P. Young, Spin glasses and random fields (World Scientific, Singapore, 1998).

[4] F. Krzakala and O. Martin, Europhys. Lett. 53, 749 (2001). T. Jörg et al., Phys. Rev. Lett. 96, $237205(2006)$.

[5] A.J. Bray and S. Feng, Phys. Rev. B 36, 8456 (1987).

[6] S. Boettcher, Europhys. Lett. 67, 453 (2004); Eur. Phys. J. B 38, 83 (2004); Phys. Rev. Lett. 95, 197205 (2005).

[7] On a cubic lattice, link percolation is $p_{c}=0.2488126(5)$. C.D. Lorenz and R.M. Ziff, Phys. Rev. E 57, 230 (1998).

[8] T. Jörg and F. Ricci-Tersenghi, Phys. Rev. Lett. 100, 177203 (2008).

[9] K. Binder, Z. Phys. B 43, 119 (1981). H.G. Ballesteros et al., Nucl. Phys. B 483, 707 (1997).

[10] Krzakala F, Montanari A, Ricci-Tersenghi F, Semerjian G and Zdeborova L, 2007 Proc. Nat. Acad. Sci. 10410318 
[11] Zdeborova L and Krzakala F, 2007 Phys. Rev. E 76031131

[12] Montanari A, Ricci-Tersenghi F and Semerjian G, 2008 J. Stat. Mech. P04004

[13] Castellani T, Krzakala F and Ricci-Tersenghi F, 2005 Eur. Phys. J. B 4799

[14] A. Hartmann and F. Ricci-Tersenghi, Phys. Rev. B 66, 224419 (2002). 